\title{
Interpretation of venous occlusion plethysmography using a nonlinear model
}

\author{
${ }^{1} \mathrm{~F}$. A. Anderson Jr. $\quad{ }^{2}$ W. W. Durgin $\quad{ }^{1} \mathrm{H}$. Brownell Wheeler \\ 'Department of Surgery, University of Massachusetts Medical School, 55 Lake Avenue North, Worcester, \\ MA 01605, USA \\ ${ }^{2}$ Department of Mechanical Engineering, Worcester Polytechnic Institute, Worcester, MA 01609, USA
}

\begin{abstract}
Venous occlusion plethysmography (VOP) is a noninvasive technique widely employed for the detection of deep-vein thrombosis. Previous reports that VOP outflow curves are closely fit by a first-order exponential suggest that venous compliance and resistance are nearly constant. Typically, however, the venous compliance function has a sigmoid shape; in addition, the resistance in a collapsing tube must increase. This paradox was resolved by the surprising finding that for realistic nonlinear compliance and resistance these nonlinearities cancel, producing a quasilinear venous outflow that approximates a simple exponential.
\end{abstract}

Keywords-Deep venous thrombosis, Theoretical model, Venous occlusion plethysmography

Med. \& Biol. Eng. \& Comput., 1986, 24, 379-385

List of symbols

\begin{tabular}{|c|c|c|}
\hline Terms & Definition & Units \\
\hline$A$ & area & $\mathrm{m}^{2}$ \\
\hline C & compliance $d V / d P$ & $m^{5} N^{-1}$ \\
\hline$D$ & diameter & $\mathrm{m}$ \\
\hline$g$ & gravitational acceleration & $\mathrm{ms} \mathrm{s}^{-2}$ \\
\hline$H_{c}$ & $\begin{array}{l}\text { height or elevation of the } \\
\text { calf }\end{array}$ & $\mathrm{m}$ \\
\hline$h_{l}$ & head loss & m \\
\hline$L$ & length & $\mathrm{m}$ \\
\hline$P_{c}$ & calf venous blood pressure & $\mathrm{Nm}^{-2}$ \\
\hline$P_{h}$ & right heart blood pressure & $\mathrm{Nm}^{-2}$ \\
\hline$Q$ & $\begin{array}{l}\text { rate of calf emptying } \\
\left(V_{c}^{\prime}=V_{b}^{\prime}\right)\end{array}$ & $\mathrm{m}^{3} \mathrm{~s}^{-1}$ \\
\hline$R$ & flow resistance $\Delta P / Q$ & $\mathrm{Nsm}^{-5}$ \\
\hline$t$ & time & $\mathbf{s}$ \\
\hline$U$ & average velocity & $\mathrm{ms} \mathrm{s}^{-1}$ \\
\hline$V_{c}$ & $\begin{array}{l}\text { segmental calf volume } \\
\text { including tissues }\left(V_{1}\right) \\
\text { and blood }\left(V_{b}\right)\end{array}$ & $\mathrm{m}^{3}$ \\
\hline$\Delta V_{\mathrm{c}}$ & $\begin{array}{l}\text { change in calf volume due } \\
\text { to venous blood volume } \\
\text { change }=V_{b}\end{array}$ & $\mathrm{~m}^{3}$ \\
\hline$V C$ & $\begin{array}{l}\text { venous capacitance }=\Delta V_{c} / V_{c} \\
\text { immediately prior to } \\
\text { release of thigh occlusion } \\
\text { pressure }\end{array}$ & per cent \\
\hline$\rho$ & density & $\mathrm{kg} \mathrm{m}^{-3}$ \\
\hline$\mu$ & dynamic (absolute) viscosity & $\mathrm{Nsm}^{-2}$ \\
\hline
\end{tabular}

Subscripts: $b$ blood $h$ heart

$c$ calf 0 initial or resting value

\section{Introduction}

VENOUS OCCLUSION plethysmography (VOP) was originally developed as a noninvasive method to assess limb blood flow (HEWLETT and VAN ZWALUWENBERG, 1909). Qualitative analysis of venous outflow curves, performed in normal subjects, was subsequently reported (LEWIS and GranT, 1925). However, evaluation of VOP curves to detect venous outflow obstruction was not reported until 1968, when a water-filled plethysmograph was employed to document the impairment of venous outflow in patients with deep venous thrombosis (DAHN and EIRIKSSON, 1968). The development of less cumbersome plethysmographic instruments has allowed VOP to become a widely employed screening method in the detection of deep venous thrombosis (WHEELER et al., 1974; HULL et al., 1981; WHEELER and ANDERSON, 1982; 1985).

VOP is performed by measuring calf volume changes in response to temporary occlusion of venous blood flow in the thigh. The basic technique of VOP is fairly standard (ANDERSON and WHEELER, 1979). The patient is tested in a supine position with the leg elevated $20-30 \mathrm{~cm}$ above heart level. A thigh cuff ( $20 \mathrm{~cm}$ wide) is inflated to a pressure of $50 \mathrm{~mm} \mathrm{Hg}$, above venous pressure but below arterial diastolic pressure. The resulting increase in calf volume is monitored until a stable plateau is achieved (typically occurring within $2 \mathrm{~min}$ ). The thigh cuff is then rapidly deflated. The presence of major venous outflow obstruction is determined by comparing the rate of venous outflow with the maximum calf volume change.

Previous attempts to model the VOP response have been based on a linear model which predicts that the venous outflow curve can be described by the maximum percentage calf volume change $\Delta V_{c} / V_{c}$ (Fig. 1), commonly referred to as the venous capacitance $(V C)$, and a single exponential time constant (STRANDNESS and SUMNER, 1975; KAY et al., 1975; JAFFRIN, 1976; SEAGAR et al., 1984). The validity of a linear VOP model is supported by the good 
fit of venous outflow curves to a single time constant in 90 normal limbs and in 33 limbs with deep-vein thrombosis (KAY et al., 1975). Similar results were recently reported from a study of three normal limbs (SEAGAR et al., 1984).

Previous reports that VOP outflow curves are closely fit by a first-order exponential suggest that venous compliance and resistance are nearly constant. However, actual venous compliance follows a sigmoid curve. In addition, the resistance in a collapsing tube must increase (CARO et al., 1978). Perhaps because VOP curves behave as though the venous system is linear, previous investigators have assumed that any nonlinearities in $R$ or $C$ must be unimportant. Realistic functions for calf compliance and venous outflow resistance are employed in the present study to produce solutions to a nonlinear model of VOP.

\section{Model of venous outflow in VOP}

To derive the system equation, a control volume is established (Fig. 2), consisting of the calf with venous blood pressure $P_{c}$, the right heart with pressure $P_{h}$ and a connecting vessel with an average flow velocity $U$. The calf

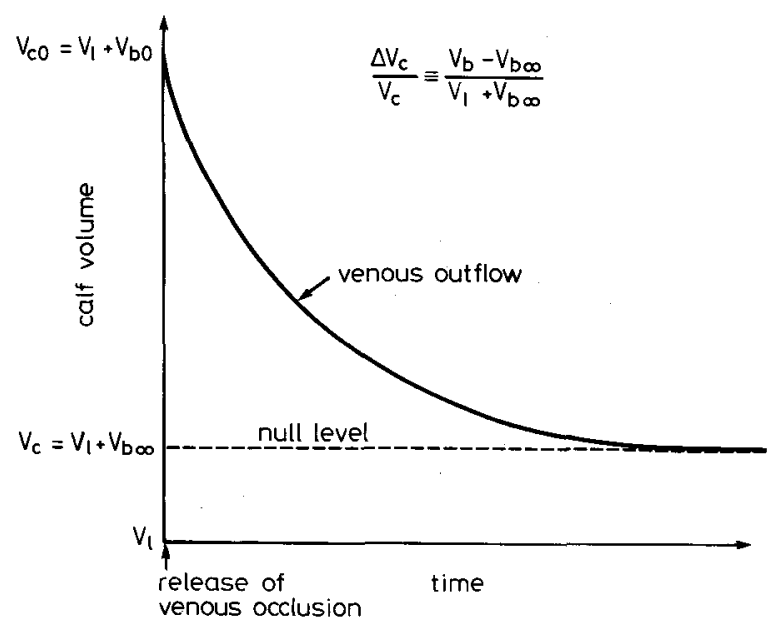

Fig. 1 Definition of the parameters of VOP venous outflow. Changes in calf blood volume are commonly expressed as a percentage of limb volume

is elevated above the heart level by a distance $H_{c}$ and the effective length of the vessel from the knee to the heart is given as $L$.

Assuming a quasi-one-dimensional flow of a viscous, incompressible fluid, the unsteady Bernoulli equation with head loss can be written as follows:

$$
P_{c}+\rho g H_{c}-P_{h}=\rho g h_{l}+\rho L \frac{\partial U}{\partial t}
$$

where the left-hand side equals the energy available in the system; $\rho g h_{l}$ equals the viscous or frictional energy loss between calf and heart and $\rho L \partial U / \partial t$ equals the additional inertial energy loss (WHITE, 1974).

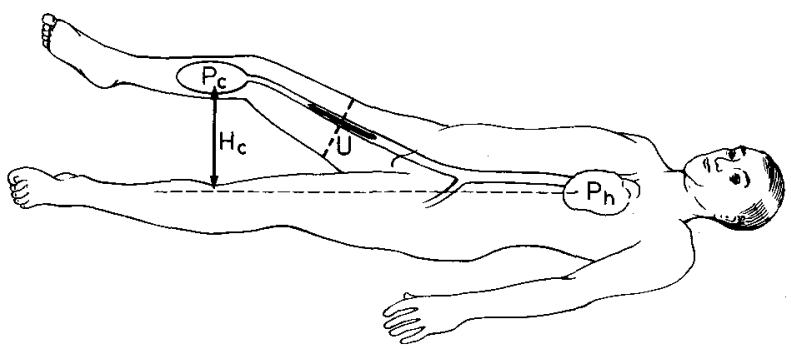

Fig. 2 Conceptual model of VOP employed to develop the theoretical solutions
The solution of eqn. 1 is simplified by excluding the inertial term (evaluated subsequently). Thus, eqn. 1 becomes

$$
P_{c}+\rho g H_{c}-P_{h}=\rho g h_{l}
$$

This equation may be solved for the change in calf volume against time, $\dot{V}_{c}$, using three basic definitions (CARO et al., 1978):

$$
\begin{array}{ll}
\text { continuity: } & \dot{V}_{c}=-Q \\
\text { resistance: } & R=\frac{d P_{c}}{Q} \\
\text { compliance: } & C=\frac{d V_{c}}{d P_{c}}
\end{array}
$$

Eqn. 2 can be expressed as

$$
d P_{c}=P_{c}-P_{h}+\rho g H_{c}=\rho g h_{l}
$$

Combining eqns. 4 and 6

$$
R=\frac{d P_{c}}{Q}=\frac{P_{c}-P_{h}+\rho g H_{c}}{Q}=\frac{\rho g h_{l}}{Q}
$$

it can now be seen that the head loss is equal to the resistance times the flow:

$$
\rho g h_{l}=R Q
$$

From the definition of compliance (eqn. 5)

$$
d V_{c}=C d P_{c}
$$

Combining eqn. 9 with continuity (eqn. 3)

$$
\frac{C d P_{c}}{d t}=-Q
$$

Since $d P_{c}=\rho g h_{l}$ (eqn. 6), eqn. 10 becomes

$$
\operatorname{C\rho g} \frac{d h_{l}}{d t}=-Q
$$

and since $\rho g h_{1}=R Q$ (eqn. 8), eqn. 11 becomes

$$
C \frac{d(R Q)}{d t}=-Q
$$

and since $\dot{V}_{c}=-Q$ (eqn. 3), eqn. 12 becomes

$$
C \frac{d\left(R \dot{V}_{c}\right)}{d t}=-\dot{V}_{c}
$$

Differentiating eqn. 13

$$
C\left(R \ddot{V}_{c}+\dot{R} \dot{V}_{c}\right)=-\dot{V}_{c}
$$

and rearranging

$$
\ddot{V}_{c}=-\frac{\dot{R}}{R} \dot{V}_{c}-\frac{\dot{V}_{c}}{R C}
$$

This is the general differential equation relating the change in calf volume during venous outflow in VOP to specific functions representing the venous outflow resistance and calf compliance.

\section{Venous compliance}

Compliance is defined as the ratio of volume change to pressure change (eqn. 5). Since the veins have thin, flexible walls, they may collapse at low transmural pressure and thus are said to be highly compliant. Whereas changes in calf volume can be determined using noninvasive techniques, including strain gauge (SGP) and impedance 
plethysmography (IPG), the blood pressure in the deep veins of the leg is not so easily determined. Previous investigators have relied on tilt-table methods (LUDBrook, 1966) or cannulation of a superficial vein (HESSE, 1970; SEAGar et al., 1984). Data for the present study were obtained from a tilt-table experiment on a normal leg. As the subject was gradually tilted from a $30^{\circ}$ foot-down to a $30^{\circ}$ foot-up orientation, venous pressure was estimated from the elevation of the calf with respect to the heart. Venous volume was determined by SGP. The sigmoid shape of these data suggests that a reasonable fit can be obtained based on the arc tangent function (Fig. 3).

$$
\begin{aligned}
V_{b}=\Delta V_{c}= & 1.645 \times 10^{-5} \text { arc tan } \\
& {\left.\left.\left[\left(P_{c}-2100\right) / 500\right)\right]+2.2 \times 10^{-5}\right) \mathrm{m}^{3} }
\end{aligned}
$$

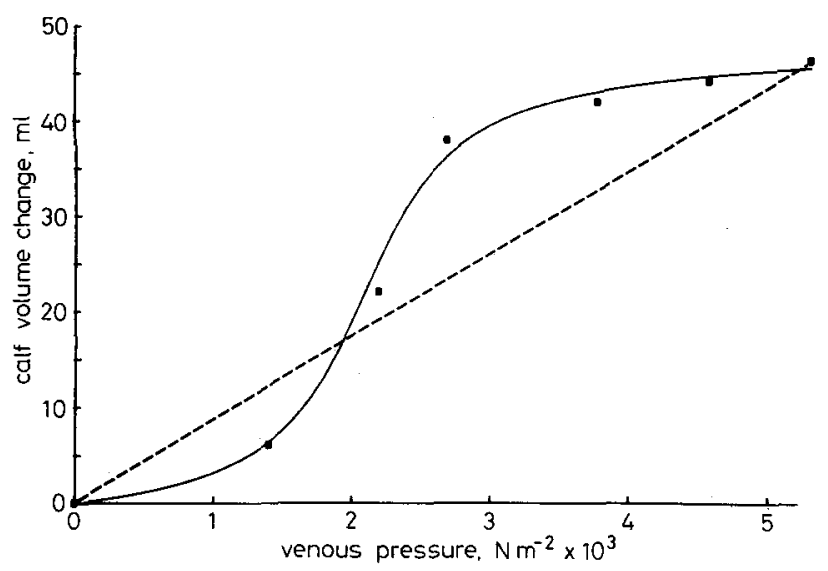

Fig. 3 Compliance of a normal human calf estimated from a tilttable experiment. The solid curve is a nonlinear empirical fit to these data. The broken line is a linear approximation of calf compliance (see text)

There is a large variation in the compliance of the calf veins, from $30 \times 10^{-9} \mathrm{~m}^{5} \mathrm{~N}^{-1}$ during the initial phase of filling following venous occlusion to less than $2.0 \times 10^{-9} \mathrm{~m}^{5} \mathrm{~N}^{-1}$ as VOP reaches a stable plateau, prior to the release of venous occlusion. Eqn. 16 can now be used to define the calf compliance as a function of $V_{b}$ using standard integral tables.

$$
C=\frac{\left(1.645 \times 10^{-5}\right) / 500}{1+\tan ^{2} \frac{\left[V_{b}-\left(2.2 \times 10^{-5}\right)\right]}{\left[1.645 \times 10^{-5}\right]}}
$$

The broken line in Fig. 3 represents a linearised estimate of the calf compliance function obtained from noninvasive data. Two points are necessary to determine a linear estimate of $C$. The first is the null pressure and volume level achieved when the calf is elevated above the heart prior to cuff inflation. In the absence of right-sided cardiac failure or other condition causing serious elevation of central venous pressure, the assumption that the resting venous pressure in the calf veins is nearly zero may be reasonable. The second reference point is equal to a fixed percentage of the applied pressure in the thigh cuff when the VOP rise is complete and a stable plateau of calf volume has been achieved. This value is a function of the width of the thigh cuff employed. It is equal to 90 per cent of applied pressure when a 16 or $20 \mathrm{~cm}$ wide cuff is employed (HESSE, 1970; ANDERSON, 1984), and 50 per cent when a 7 or $10 \mathrm{~cm}$ cuff is used (HeSse, 1970; SEAGAR et al., 1984). If a standard cuff and occlusion pressure are employed, the distal venous pressure will be nearly constant at maximum venous filling $(V C)$. A noninvasive estimate of the 'linearised' calf compliance can now be determined as a function of $V C$ alone.
Assuming a $V C$ equal to $3 \cdot 2$ per cent and calf volume $V_{c}=1375 \mathrm{~cm}^{3}$, then $\Delta V_{c 0}=V_{b 0}=(3.2$ per cent $)$ $(1375 \mathrm{ml})=44 \mathrm{ml}\left(=4.4 \times 10^{-5} \mathrm{~m}^{3}\right)$. If cuff pressure is $50 \mathrm{~mm} \mathrm{Hg}$, then $P_{c}=45 \mathrm{~mm} \mathrm{Hg}$. Assuming a resting venous pressure of $5 \mathrm{~mm} \mathrm{Hg}$, then $\Delta P_{c}=40 \mathrm{~mm} \mathrm{Hg}$ or $5320 \mathrm{~N} \mathrm{~m}^{-2}$.

Thus, for a normal limb,

$$
C=\frac{\Delta V_{c}}{\Delta P_{c}}=\frac{4.4 \times 10^{-5}}{5320}=8.27 \times 10^{-9} \mathrm{~m}^{5} \mathrm{~N}^{-1}
$$

These approximations of the actual nonlinear calf compliance and the linearised constant calf compliance can now be employed to demonstrate the character of the corresponding linear and nonlinear theoretical solutions.

\section{Venous outflow resistance}

Prior to venous outflow, the veins distal to the thigh occlusion cuff are cylindrical. Following the release of cuff pressure, the veins partially collapse forming an elliptical cross-section. It is possible to document these normal variations in vein geometry by means of an ultrasonic cross-sectional image of the deep veins during VOP outflow. The veins do not collapse completely, probably due to resting venous blood flow. Measurements from ultrasonic images show that the final area is approximately 70 per cent of the initial circular cross-section $(b / a=0 \cdot 40)$. Thus a normal vein can be viewed as an elliptical tube whose axial ratio $(b / a)$ ranges from 1.0 to 0.4 during venous outflow (ANDERSON, 1984).

Assuming laminar flow, venous outflow resistance for a long, elliptical vessel can be predicted from Poiseuille's Law (CARo et al., 1978). Venous resistance will increase by a factor of approximately two as the vein collapses from $b / a=1.0$ to 0.4

The initial flow resistance in the deep veins can now be estimated from Poiseuille's Law:

$$
R=\frac{\Delta P}{Q}=\frac{128 \mu L}{\pi D^{4}}
$$

Assuming a constant blood viscosity of $4 \times 10^{-3} \mathrm{~N} \mathrm{~s} \mathrm{~m}^{-2}$,

$$
R=0 \cdot 16 \frac{L}{D^{4}}
$$

Thus, in laminar flow, resistance is a function of geometry alone. In adults, the diameter of the popliteal vein $D$ averages about $0.78 \mathrm{~cm}$. $L$ is taken as $50 \mathrm{~cm}$, the approximate distance from the knee to the iliac vein (ANDERSON, 1984). Based on these values of $D$ and $L$, eqn. 19 predicts a resistance of $2.16 \times 10^{7} \mathrm{~N} \mathrm{~s} \mathrm{~m}^{-5}$ in the deep veins of the thigh. Additional flow resistance must be included for the multiple parallel venous channels of the calf. Assuming that there are three main deep veins in the calf that channel the flow to the popliteal vein, each with $D=0.3 \mathrm{~cm}$ and $L=20 \mathrm{~cm}$, then by eqn. $19, \quad R_{1}=R_{2}=R_{3}=3.951$ $\times 10^{8} \mathrm{Ns} \mathrm{m}^{-5}$. The total calf component of flow resistance is found as

$$
\frac{1}{R_{c}}=\frac{1}{R_{1}}+\frac{1}{R_{2}}+\frac{1}{R_{3}}=\frac{3}{R_{1}}
$$

and $R_{c}=R_{1} / 3=1.317 \times 10^{8} \mathrm{~N} \mathrm{~s} \mathrm{~m}^{-5}$.

This is more than five times the resistance estimated in the normal proximal deep veins. Thus some care must be taken when evaluating VOP as a lumped-element system. While the calf veins may be modelled as an equivalent compliance element, the individual calf veins are the main source of venous outflow resistance in the absence of major venous outflow obstruction. The total resistance of 
the normal leg veins can now be estimated as $1.533 \times 10^{8} \mathrm{Ns} \mathrm{m}^{-5}$, the sum of the calf and proximal resistances (ANDERSON, 1984).

\section{Theoretical model results}

\subsection{Constant compliance and resistance}

Solution of this special case of eqn. 15 requires the assumption of a constant resistance for a given degree of venous obstruction (laminar flow and constant geometry) and linear compliance $(C=$ constant $)$. This can be pictured as a first-order system, similar to a balloon, consisting of one equivalent storage volume (the veins of the calf) connected to a single equivalent flow resistance (the proximal deep veins). This is also the solution for the 'Windkessel' model of venous emptying that has been employed by previous investigators to predict VOP behaviour.

Since $R$ is held constant, $\dot{R}=0$ and eqn. 15 becomes

$$
\ddot{V}_{c}=-\frac{\dot{V}_{c}}{R C}
$$

This expression can now be integrated, giving a simple exponential solution:

$$
\frac{\Delta V_{c}}{V_{c}}=V C e^{-t / R C}
$$

Applying the previously estimated values, $R=$ $1.533 \times 10^{8} \mathrm{~N} \mathrm{~s} \mathrm{~m}^{-5}$

$$
\begin{gathered}
C=8.27 \times 10^{-9} \mathrm{~m}^{5} \mathrm{~N}^{-1} \text { and } V C=3.2 \text { per cent, } \\
\frac{\Delta V_{c}}{V_{c}}=3.2 e^{-t / 1.27 \mathrm{~s}}
\end{gathered}
$$

This result is plotted in Fig. 4.

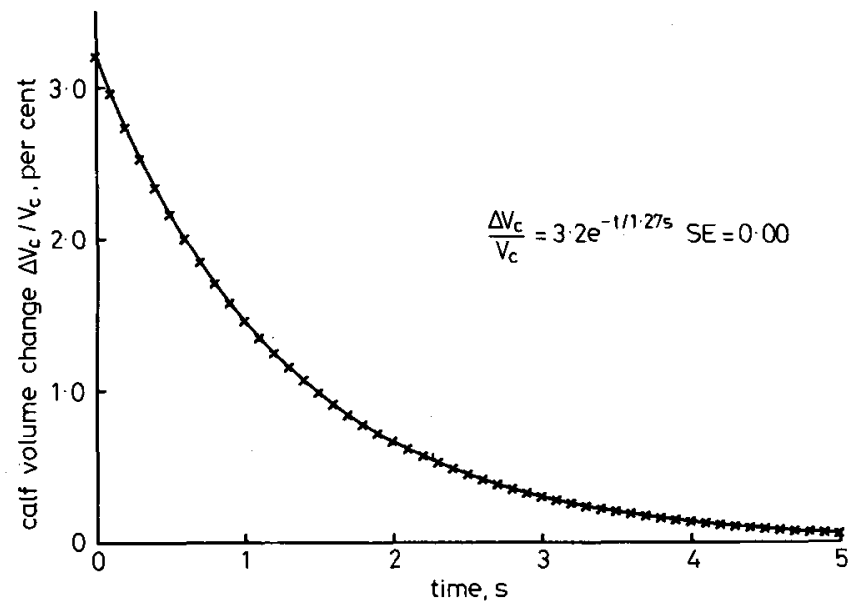

Fig. 4 A theoretical solution for VOP outflow in a normal leg assuming constant resistance and compliance. The solid curve is an RMS first-order exponential fit to this theoretical result

\subsection{Nonlinear resistance}

If resistance is not constant during venous outflow (VO), then blood flow must be turbulent and/or vessel geometry must vary. The Reynolds number during VOP is too low for turbulence to occur (ANDERSON, 1984). Thus, if $R$ is non-constant, it must vary as a result of changes in vessel geometry alone. The popliteal vein has been observed to partially collapse during VO. A change of $b / a=1.0$ to approximately 0.4 (or an area reduction from 100 per cent to 70 per cent of the initial circular cross-section) was seen in ultrasonic images of the popliteal vein during VO. The theoretical relationship between $R$ and $b / a$ for an elliptical tube predicts that $R$ will increase by a factor of approximately two when $b / a$ is in this range.

Assuming $R$ increases linearly during VO,

$$
\frac{R}{R_{0}}=-\frac{V_{b}}{V_{b 0}}+2 \cdot 0
$$

then

$$
R=-\frac{R_{0}}{V_{b 0}} V_{b}+2 R_{0}
$$

and

$$
\dot{R}=-\frac{R_{0}}{V_{b 0}} \dot{V}_{b}
$$

Thus, substituting these results into eqn. 15 ,

$$
\ddot{V}_{c}=\frac{\dot{V}_{c} \dot{V}_{c}}{2 V_{b 0}-V_{b}}-\frac{V_{b 0} \dot{V}_{c}}{R_{0} C\left(2 V_{b 0}-V_{b}\right)}
$$

Assuming $V_{b 0}=4.4 \times 10^{-5} \mathrm{~m}^{3}, C=8.27 \times 10^{-9} \mathrm{~m}^{5} \mathrm{~N}^{-1}$ and $R_{0}=1.533 \times 10^{8} \mathrm{Nsm}^{-5}$, and since $\ddot{V}_{c}=\ddot{V}_{b}$ and $\dot{V}_{c}=\dot{V}_{b}$, eqn. 26 becomes

$$
\ddot{V}_{b}=\frac{\dot{V}_{b} \dot{V}_{b}}{\left(8.8 \times 10^{-5}\right)-V_{b}}-\frac{\left(4.4 \times 10^{-5} \dot{V}_{b}\right)}{1.27\left[\left(8.8 \times 10^{-5}\right)-V_{b}\right)} \mathrm{m}^{3}
$$

The solution was found by the Runge-Kutta method of numerical integration (CARNAHAN et al., 1969), assuming $\dot{V}_{b 0}=V_{b 0} / R C=-2 \cdot 39 \times 10^{-5} \mathrm{~m}^{3} \mathrm{~s}^{-1}$. Resulting values of $V_{b}$ against time are divided by an assumed calf volume, $V_{c}=1.375 \times 10^{-3} \mathrm{~m}^{3}$, to give a plot in the standard form (see Fig. 5).

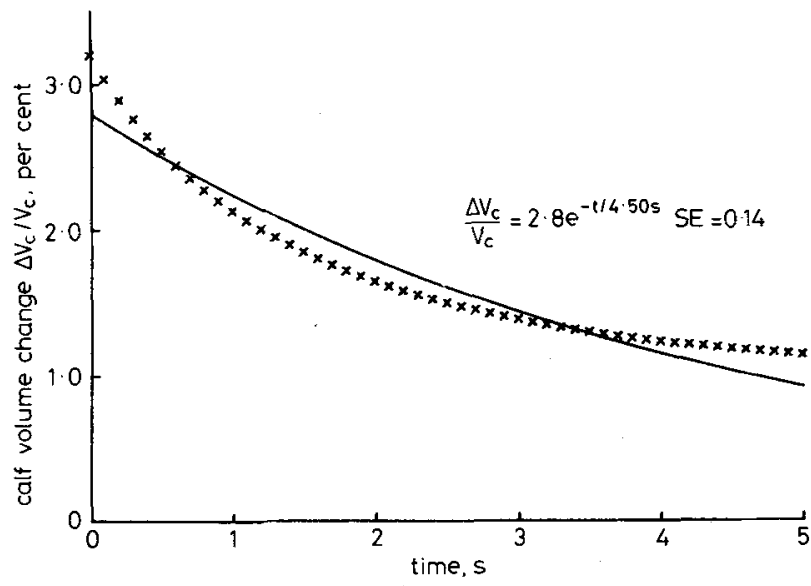

Fig. 5 A theoretical solution for VOP outfow in a normal leg, assuming variable resistance and constant compliance. The solid curve is an RMS first-order exponential fit to this theoretical result

\subsection{Nonlinear compliance}

The effect of nonlinear compliance on VO curves can be predicted by expressing $C$ as a function of calf venous volume $V_{b}$ when resistance is constant. Substituting $R=1.533 \times 10^{8} \mathrm{Nsm}^{-5}$ and $C$ from eqn. 17 into the general solution (eqn. 15) gives,

$$
\ddot{V}_{b}=-0.20\left[1+\tan ^{2}\left\{\left(6.08 \times 10^{4}\right) V_{b}-1.337\right\}\right) \dot{V}_{b}
$$

Assuming $\quad V_{b 0}=4.4 \times 10^{-5} \mathrm{~m}^{3} \quad$ and $\quad \dot{V}_{b 0}=-2.39$ $\times 10^{-5} \mathrm{~m}^{3} \mathrm{~s}^{-1}$, this equation was solved by the RungeKutta method. Resulting values of $V_{b}$ against time are divided by an assumed calf volume, $V_{c}=1.375 \times 10^{-3} \mathrm{~m}^{3}$, to give a plot in the standard form (see Fig. 6). 


\subsection{Combined nonlinear $R$ and $C$}

Viewed individually, compliance and resistance changes during venous outflow produce significant changes in the shape of VO curves, particularly in limbs without major venous outflow obstruction. A complete solution will now be obtained using realistic nonlinear functions for both $R$ and $C$. Eqn. 15 becomes

$$
\begin{aligned}
\ddot{V}_{b}= & \frac{\dot{V}_{b} \dot{V}_{b}}{\left(8 \cdot 8 \times 10^{-5}\right)-V_{b}} \\
& -\frac{\left(4 \cdot 4 \times 10^{-5}\right)\left(1+\tan ^{2}\left(6.08 \times 10^{4}\right) V_{b}-1.337\right) \dot{V}_{b}}{5 \cdot 04\left\{\left(8 \cdot 8 \times 10^{-5}\right)-V_{b}\right\}}
\end{aligned}
$$

Eqn. 29 has been solved using the Runge-Kutta method. Resulting values of $V_{b}$ against time are divided by an assumed calf volume $V_{c}=1.375 \times 10^{-3} \mathrm{~m}^{3}$ to give a plot in the standard form (see Fig. 7).

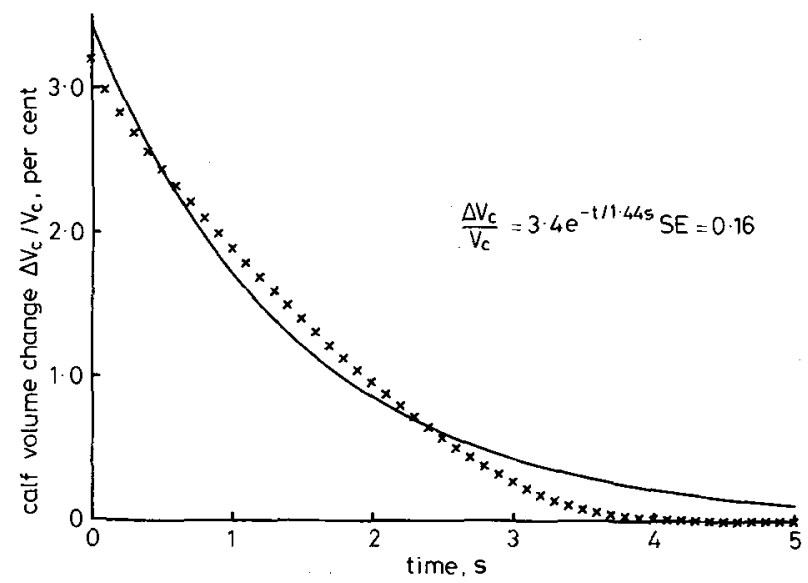

Fig. 6 A theoretical solution for VOP outflow in a normal leg assuming constant resistance and nonlinear compliance. The solid curve is an RMS first-order exponential fit to this theoretical result

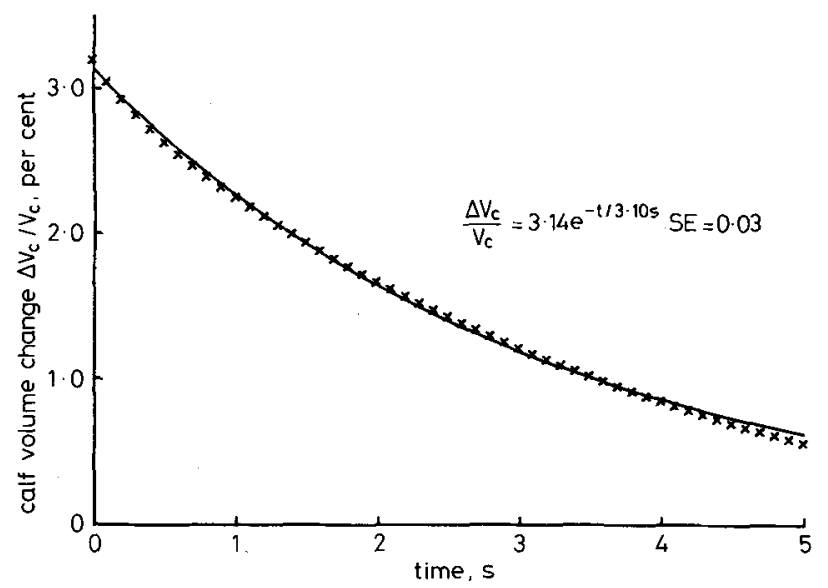

Fig. 7 A theoretical solution for VOP outflow in a normal leg assuming nonlinear resistance and compliance. The solid curve is an RMS first-order exponential fit to this theoretical result

\section{Significance of inertia}

The unsteady Bernoulli Equation (eqn. 1) must be solved to evaluate the effect of inertia on the normal VOP outflow curve. The simplest approach is to assume a constant vessel area $A$ and length $L$, where $U=A / L$. The solution parallels the development of eqn. 15 , resulting in a second-order differential equation of the form

$$
\ddot{Q}+2 n \dot{Q}+p^{2} Q=0
$$

where $n=R A / 2 L$ and $p^{2}=A / C L$. Assuming $R=$ $1.533 \times 10^{8} \mathrm{Ns} \mathrm{m}^{-5}, C=8.27 \times 10^{-9} \mathrm{~m}^{5} \mathrm{~N}^{-1}, L=0.5 \mathrm{~m}$, $A=4.8 \times 10^{-5} \mathrm{~m}^{2}$ and $V C=3.2$ per cent, then $n=7.35$ and $p^{2}=11 \cdot 61$. Solving for the roots of eqn. 30 ,

$$
\frac{\Delta V_{c}}{V_{c}}=3 \cdot 2\left(1 \cdot 07 e^{-t / 1 \cdot 19}-0.06 e^{-t / 0 \cdot 07}\right)
$$

This solution, together with the linear solution excluding inertial terms (Fig. 4), is plotted in Fig. 8. This approximation of the inertial contribution to venous outflow in a normal limb predicts a small effect largely limited to a slight delay in venous emptying in the first $0.2 \mathrm{~s}$ following the release of occlusion. It is interesting to relate this theoretical prediction to human data where a similar delay occurs between the release of cuff occlusion and the fall of the VOP outflow curve.

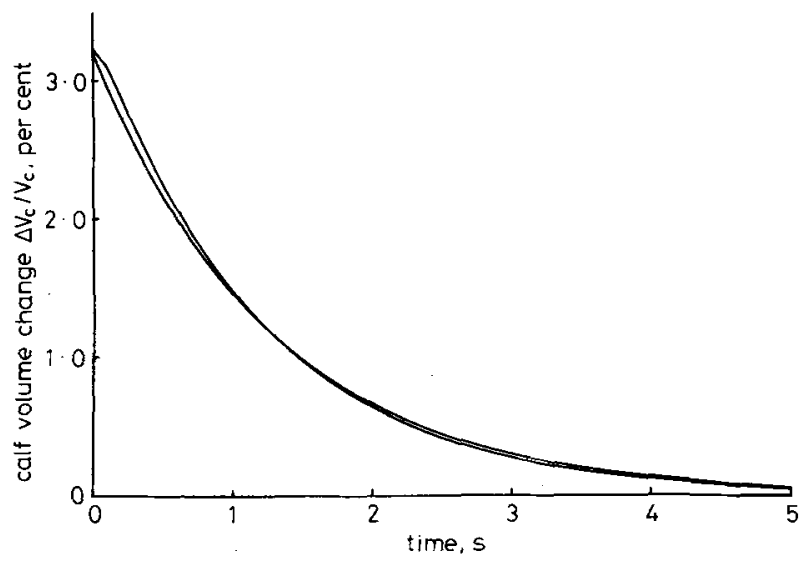

Fig. 8 Comparison of linear (bottom curve) and inertial (top curve) theoretical solutions for VOP outflow in a normal leg

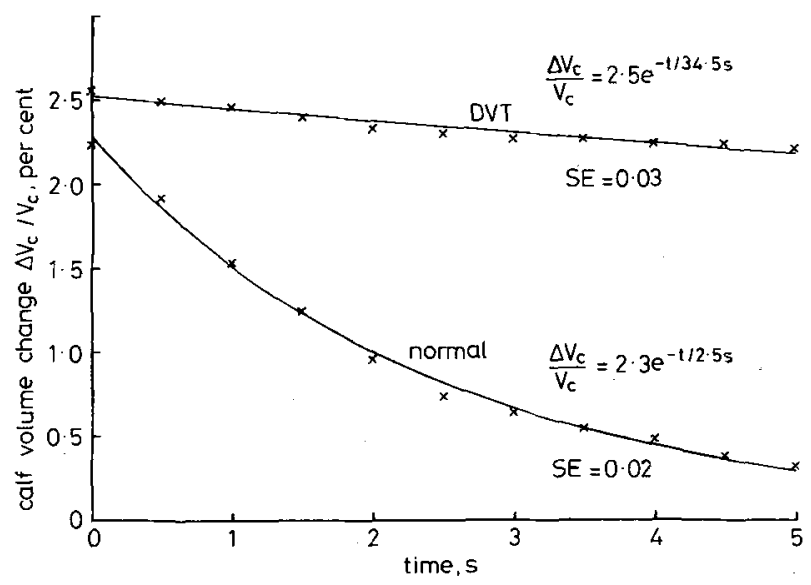

Fig. 9 Representative VOP outflow data obtained in 126 patients. The solid curves are an RMS first-order exponential fit to these data

\section{VOP data from humans}

VOP curves were obtained using the IPG in 126 legs of 100 patients with suspected DVT. Venograms were performed on all 126 legs and confirmed the presence of acute major DVT in 33 limbs. Venous outflow was hand digitised for a period of $5 \mathrm{~s}$ following the release of thigh occlusion pressure. Eleven points were recorded at equal time intervals. These data were entered into a computer, and a least-squares exponential regression was performed. The accuracy of fit was quantified by expressing the RMS standard error of estimation (SE) as a percentage of the initial magnitude of calf filling $V C$.

Additional VOP testing was performed using IPG and SGP simultaneously in 100 legs of 50 healthy volunteers, including eight children. VOP data were simultaneously 
recorded on a dual-channel thermal recorder and a digital computer using an analogue-to-digital interface at a sampling rate of $16 \mathrm{~Hz}$. All 100 pairs of SGP/IPG venous outflow curves were fit to a first-order exponential. The RMS standard error (normalised to $V C$ ) averaged 0.016 in 126 legs of patients and was 0.011 (IPG), 0.010 (SGP) in 100 legs of healthy volunteers. In 33 legs with venographically confirmed acute DVT extending proximal to the $\mathrm{knee}$, the average time constant was $5 \cdot 6 \mathrm{~s}$. The corresponding value in 100 normal legs was $1.7 \mathrm{~s}$ by SGP and $1.9 \mathrm{~s}$ by IPG. A typical patient tracing is shown in Fig. 9 and volunteer data in Fig. 10. The $V C$ is plotted as a function of the time constant of venous emptying in Fig. 11 for IPG results from 100 normal limbs and 33 limbs with DVT.

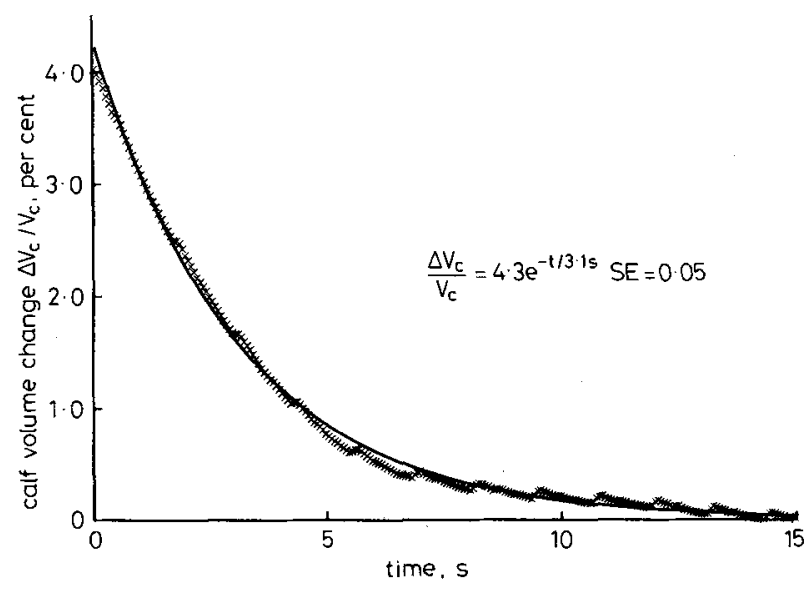

Fig. 10 Representative VOP outflow data obtained from 100 legs of healthy volunteers. The solid curve is an RMS firstorder exponential fit to these data

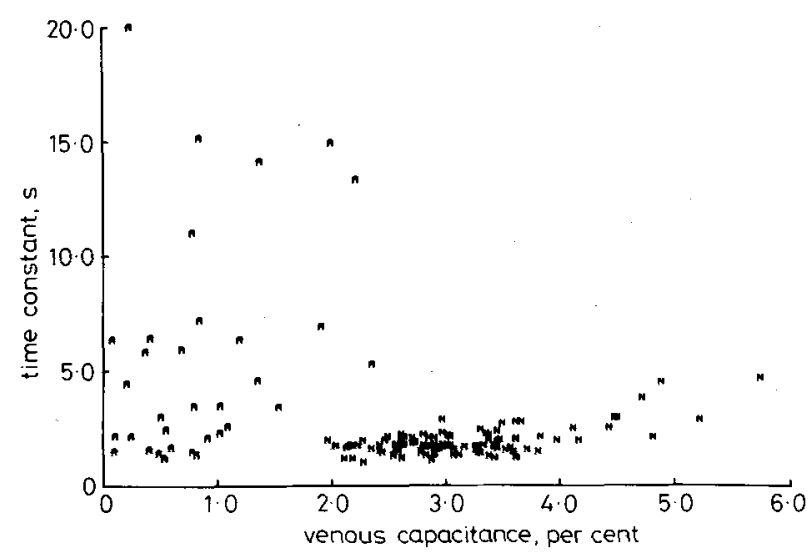

Fig. 11 Venous capacitance $V C$ is plotted as a function of the time constant of venous outflow, as measured by IPG, in 100 normal limbs $(N)$ as well as in 33 limbs with acute proximal $D V T(A)$

\section{Discussion}

Evaluation of VOP data in 226 human limbs confirmed previous reports that venous outflow curves follow the form of a first-order exponential function. This result suggests a linear system in which both venous outflow resistance and calf compliance are nearly constant during VO. This seems incompatible with the recognised nonlinearity of calf venous compliance. A resolution of this paradox has been presented based on the solution of a nonlinear model of VOP. The theoretical solution obtained by using a realistic nonlinear calf compliance function (assuming constant venous outflow resistance) does not follow the form of a simple exponential (Fig. 6); however, when reasonable nonlinear relationships for both calf compliance and venous outflow resistance are employed, the resulting curve is surprisingly similar to a simple exponential (Fig. 7).
The initial resistance of the unobstructed deep veins of the leg was calculated as $1.533 \times 10^{8} \mathrm{Ns} \mathrm{m}^{-5}$ from Poiseullie's Law and estimates of vein dimensions. It was further estimated that the venous flow resistance would double in the late phase of VO as the veins partially collapse. Thus the corresponding final venous resistance would be $3.066 \times 10^{8} \mathrm{~N} \mathrm{~s} \mathrm{~m}^{-5}$.

The 'linearised' or average venous outflow resistance can be estimated from a simple exponential fit to VO curves by dividing the average $R C$ time constant, $1.7 \mathrm{~s}$ (SGP) or $1.9 \mathrm{~s}$ (IPG), by the average 'linearised' calf compliance, $8.27 \times 10^{-9} \mathrm{~m}^{5} \mathrm{~N}^{-1}$, giving an average resistance of approximately $2.0 \times 10^{8} \mathrm{~N} \mathrm{~s} \mathrm{~m}^{-5}$. Thus the theoretical prediction that $R$ will vary from $1.533 \times 10^{8}$ to $3.066 \times 10^{8} \mathrm{~N} \mathrm{sm}^{-5}$ is in reasonable agreement with this average 'linearised' estimate of venous outflow resistance obtained in 100 normal limbs. A similar estimate calculated for 33 legs with venographically proven deep vein thrombosis and based on an average $R C$ time constant of $5.6 \mathrm{~s}$ gives an average venous outflow resistance of approximately $7.0 \times 10^{8} \mathrm{~N} \mathrm{~s} \mathrm{~m}^{-5}$.

Poiseulle's Law was applied to estimates of flow resistance in individual limbs to predict the extent of venous obstruction (see eqn. 19). However, comparison of these data with venographic estimates of thrombus size suggests that Poiseuille's Law is not applicable to the typical geometry of venous outflow obstruction due to DVT. This geometry may be more accurately compared to an orifice. The flow regime is laminar, however, and energy losses will not increase with $Q^{2}$, as predicted for turbulent flow. Therefore the actual energy loss relationship must be determined from experimental data.

Despite the development of reasonable nonlinear functions for venous outflow resistance and calf compliance, the theoretical model remains a 'lumped element' representation of a complex system. Several techniques, including ultrasonic imaging, may be employed to better define the dynamic behaviour of the deep veins along their entire length. In addition, a more extensive investigation of vein compliance is needed, particularly as a function of such factors as limb elevation. These data could be employed to develop a more complete theoretical model by taking into account the distributed nature of venous resistance and compliance. It would be particularly interesting to model the effect of a blood pressure cuff in compressing, and then releasing, a large portion of the femoral vein.

Although simple by comparison with the complex nature of venous dynamics, the current study has modelled realistic nonlinear functions for calf compliance and venous outflow resistance. The quasilinear response predicted by this model appears to explain previous reports that VOP venous outflow curves are closely approximated by a linear (first-order exponential) function. These findings may be useful in the noninvasive estimation of venous outflow resistance and in predicting the location and severity of DVT.

Acknowledgments-The authors are indebted to Ms Kathryn Graham and Ms Lauren Stratouly for their assistance in computer programming as well as in other significant aspects of this research.

\section{References}

ANDERSON, F. A. Jr. and WheELER, H. B. (19/9) venous occiusion plethysmography for the detection of venous thrombosis. Med. Instrum., 13, 350-354.

ANDERSON, F. A. Jr. (1984) Quantification of the degree of venous outflow obstruction from venous occlusion plethysmography. 
Doctoral Dissertation. Worcester Polytechnic Institute, Worcester, Massachusetts, USA.

Carnahan, B., Luther, H. A. and Wilkes, J. O. (1969) Applied numerical methods. John Wiley \& Sons Inc., New York, 363 366.

Caro, C. G., Pedley, R. C. and Seed, W. A. (1978) The mechanics of the circulation. Oxford University Press, New York.

DAHN, I. and EIRIKSSON, E. (1968) Plethysmographic diagnosis of deep venous thrombosis of the leg. Acta. Chir. Scand., Suppl $398,33-42$.

Hesse, B. (1970) Venous pressure changes during congestion. Acta. Med. Scand., 188, 447-449.

Hewlett, A. W. and VAN ZWALUwenburG, J. G. (1909) The rate of blood flow in the arm. Heart, 1, 87-97.

Hull, R., Hirsh, J., Sackett, D. L., Taylor, D. W., Carter, E., TurPie, A. G. G., Zielinsky, A., Powers, P. and Gent, M. (1981) Replacement of venography in suspected venous thrombosis by impedance plethysmography and ${ }^{125}$ I-fibrinogen leg scanning. Ann. Intern. Med., 94, 12-15.

JAFFRIN, M. Y. (1976) Rationale of plethysmographic detection of deep venous thrombosis. Proc. 29th Ann. Conf on Eng. in Med. and Biol., 18, 317.

Kay, S. M., Peura, R. A., Wheeler, H. B. and Anderson, F. A Jr. (1975) Quantification of the venous outflow portion of an impedance phlebogram. Proc. 3rd Ann. New England Bioeng. Conf., Pergamon Press, New York, 159-170.

LEWIS, T. and GRANT, R. (1925) Observation upon reactive hyperemia in man. Heart, 12, 73-120.

LUDBRoOK, J. (1966) Aspects of venous function in the lower limbs. Charles C. Thomas, Springfield, Illinois.

Seagar, A. D., GibBs, J. M. and Davis, F. M. (1984) Interpretation of venous occlusion plethysmographic measurements using a simple model. Med.\& Biol. Eng. \& Comput., 22, 12-18.

Strandess, D. E. and Sumner, D. S. (1975) Hemodynamics for surgeons. Grune \& Stratton, New York, 496-500.

Wheeler, H. B., O'Donnell, J. A., ANDerson, F. A. Jr. and BENEDICT, K. Jr. (1974) Occlusive impedance plethysmography: a diagnostic procedure for venous thrombosis and pulmonary embolism. Progr. Cardiovasc. Dis., 17, 199-205.

WheEler, H. B. and Anderson, F. A. Jr. (1982) Impedance plethysmography. In Manual for the vascular diagnostic labor atory. YAO, S. J. T. and Kempozinski, R. F. (Eds.), Year Book Medical Publishers, Chicago, 277-304.

WheEler, H. B. and ANDERson, F. A. Jr. (1985) The diagnosis of venous thrombosis by impedance plethysmography. In Noninvasive diagnostic techniques in vascular disease, 3rd. ed. BERNSTEIN, E. F. (Ed.), C.V. Mosby Co., St. Louis, 755-765.

WHITE, F. M. (1974) Viscous fluid flow. McGraw-Hill, New York.

\section{Authors' biographies}

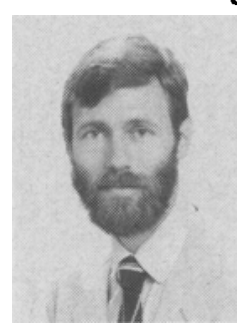

Frederick A. Anderson Jr. was born in Worcester, Massachusetts on the 3rd March 1948. He received the B.S. degree in Mechanical Engineering and B.A. in Psychology from Tufts University, Medford, Massachusetts in 1971, and his M.S. (1975) and Ph.D. (1984) in Biomedical Engineering from the Worcester Polytechnic Institute, Massachusetts. $\mathrm{He}$ is currently a Research Assistant Professor in the Department of Surgery at the University of Massachusetts Medical School, at Worcester, where he is conducting research on the noninvasive assessment of peripheral vascular disease.

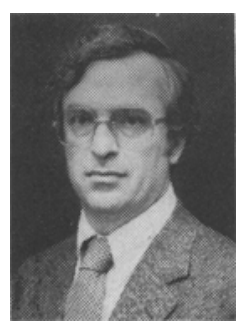

Dr. William W. Durgin was born in Framingham, Massachusetts, in 1942. He received the Sc.B. degree from Brown University in 1964, the M.S. from the University of Rhode Island in 1966 and the Ph.D. from Brown in 1970, all in Mechanical Engineering. He was an assistant professor at Florida until 1972 when he joined Worcester Polytechnic Institute's faculty of Mechanical Engineering and the Alden Research Laboratory. At present, he is the G.I. Alden Professor of Engineering and directs the Fluid Dynamics and Thermal Processes Laboratory. His current research activities include unsteady fluid mechanics, fluid-structure interaction and microgravity fluid dynamics.

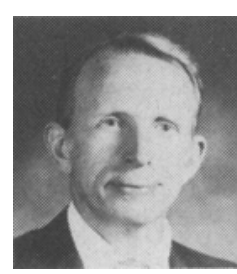

H. Brownell Wheeier was born in Louisville, KY in 1929. He received the B.S. degree from Vanderbilt University, Nashville, TN, and the M.D. degree from Harvard Medical School, Cambridge, MA, in 1952. After surgical training at the Peter Bent Brigham Hospital, he remained on the faculty of Harvard Medical School. He also served on the staff of the hospital where he became Chief of Surgery in 1961 and Chief of Staff in 1968. In 1971, he became Professor and Chairman of the Department of Surgery at the University of Massachusetts Medical School, Worcester, MA. His research interests are in developing noninvasive techniques for the evaluation of the peripheral circulation. He is a member of the Society of The University Surgeons and the American College of Surgeons. 